CERN-TH/97-207

\title{
Liapunov Exponents and the Reversibility of Molecular Dynamics Algorithms
}

\author{
Chuan Liu \\ Department of Physics \\ Peking University \\ Beijing, 100871, P.R. China \\ Andreas Jaster \\ Institut f. Theoretische Physik \\ der Technischen Universität Braunschweig \\ Mendelssohnstr. 3 \\ D-38106 Braunschweig, Germany \\ Karl Jansen \\ CERN-Theory Division \\ CH-1211 Genève 23, Switzerland
}

\begin{abstract}
We study the phenomenon of lack of reversibility in molecular dynamics algorithms for the case of Wilson's lattice QCD. We demonstrate that the classical equations of motion that are employed in these algorithms are chaotic in nature. The leading Liapunov exponent is determined in a range of coupling parameters. We give a quantitative estimate of the consequences of the breakdown of reversibility due to round-off errors.
\end{abstract}




\section{Introduction}

Ever since Wilson's formulation of QCD for a Euclidean lattice, numerical simulations have played an important role in non-perturbative investigations of physics concerning the strong interaction. When performing numerical simulations of lattice QCD, especially with dynamical fermions, one usually relies on molecular dynamics kind of algorithms such as the Hybrid Monte Carlo (HMC) [1] - the most popular one or the Kramers equation algorithm [2, 3].

A major ingredient of these algorithms is the -numerical-integration of a set of non-linear differential equations, the classical equations of motion. The non-linearity of these equations may give rise to the suspicion that the equations of motion may behave like those of a chaotic classical dynamical system. Indeed, it was first shown in [3] and later confirmed in [4, 5, 6] that the equations of motion used in the HMC algorithm, when applied to QCD, are chaotic in nature. This amusing but, at first sight, academic observation has an important consequence for any practical application of such kind of algorithms.

The exactness of the molecular dynamics kind of algorithms is guaranteed by the -sufficient- detailed balance condition. For this condition to hold, the equations of motion for the system, which govern the classical motion, are to be reversible. However, as noticed some time ago [7], this reversibility condition is violated due to round-off errors occurring in the numerical integration of the equations of motion. The fact that the equations of motion are chaotic in nature now means nothing else but that the round-off errors get magnified exponentially along the integration of the equations of motion with a positive Liapunov exponent $\nu$. This by now well-established phenomenon [3, 4, 15, 6, 8] therefore leads to rounding-error effects much larger than might naively be expected. Because of these violations of the reversibility condition, the algorithms are no longer exact from a principle point of view. Of course, the question remains at what quantitative level the reversibility violation manifests itself in physical observables.

In this paper, we provide a detailed study of the Liapunov exponents for the case of QCD and determine them in a range of values for the couplings of the theory. A short account of our results has appeared in [8]. The dependence of the leading Liapunov exponent on the coupling constants allows us to estimate where, in the coupling parameter 
space, we may expect a stronger or weaker exponential amplification of rounding-error effects. We then try to estimate how much the reversibility violation affects physical observables such as the plaquette, the Polyakov line, plaquette correlation functions and the scalar density. Finally we emphasize the special role of the acceptance Hamiltonian with respect to the rounding-error effects.

Although we will discuss these effects here for the case of lattice QCD, we think that similar problems may also arise in different fields where molecular dynamics algorithms are used, e.g. in fluid dynamics. There again it might be that the equations of motion used resemble those of a chaotic, classical, dynamical system with a positive Liapunov exponent. In some cases, as in part of the QCD simulations presently done, computers with 32-bit arithmetic are used. For these case, especially, it is desirable to know whether rounding-errors have a noticeable and significant influence on the values of physical observables. In addition, to speed up the numerical simulations, even on 64-bit arithmetic machines the time-consuming part of the computational work is sometimes performed in 32-bit arithmetic, for which case one would again be interested in the rounding-error effects.

\section{Molecular dynamics equations for lattice QCD}

Let us briefly sketch the HMC algorithm that is used for simulations of lattice QCD. Consider first for simplicity a quantum mechanical system quantized by Feynman's path integral prescription and specified by its action $S(q)$, where $q$ denotes a path of the quantum mechanical particle. In order to evaluate Feynman's path integral numerically, one sets the system on a Euclidean discrete time lattice. The task is then to generate classical paths $q$ that are distributed according to the Boltzmann distribution $e^{-S}$.

One way to proceed is to use a method that resembles those used in molecular dynamics simulations. To this end one introduces an additional, fictitious so-called Monte Carlo time $\tau$ and corresponding momenta $p$. The HMC algorithm then works as follows.

One starts from some given path $q_{i}$ on the discrete time lattice and generates initial momenta $p_{i}$ from a Gaussian distribution of unit variance and zero mean. From this initial set $\left(q_{i}, p_{i}\right)$ one computes an initial classical Hamiltonian $H\left(p_{i}, q_{i}\right)$, where $H=\frac{1}{2} p^{2}+S(q)$. The fictitious time 
evolution of the momenta and the paths is now given by the following set of coupled first-order differential equations:

$$
\dot{p}=-\frac{\delta H}{\delta q}, \dot{q}=p .
$$

The time derivatives in eqs. (1) are to be understood with respect to the fictitious Monte Carlo time. The numerical integration of the equations given in eqs. (11) is performed by using a discretized form of eqs. (11). In practice one uses a leap-frog integration scheme, using $N_{m d}$ integration steps of size $\delta \tau$ in order to integrate from fictitious time 0 to some value $\tau$. Such an integration procedure is called a trajectory of length $\tau$.

In order to generate the desired Boltzmann distribution and to account for the discretization errors of the integration procedure, the new momenta $p_{f}$ and coordinates $q_{f}$ obtained after the integration of the equations of motion are only accepted with a certain probability $P$, $P=\min (1, \exp \{-\Delta H\})$ with $\Delta H=H\left(p_{f}, q_{f}\right)-H\left(p_{i}, q_{i}\right)$. The Hamiltonians that enter this Metropolis step are called the acceptance Hamiltonians. The desired distribution is now obtained by repeating this procedure a sufficiently large number of times. The above prescription ensures the detailed balance condition, and the resulting algorithm is hence exact in the sense that it indeed converges to the anticipated Boltzmann distribution.

The heart of the algorithm sketched above is the integration of the classical equations of motion eqs. (I). This part is reminiscent of techniques used in molecular dynamics algorithms. Quite often the equations of motion derived from the problem under consideration are nonlinear and may as such correspond to those characterizing a chaotical dynamical system.

Let us now come to our example of lattice $Q C D$, which is a relativistic field theory defined in 4-dimensional Euclidean space-time. To be specific, we will focus our discussion on the equations that arise in the simulations of QCD in Wilson's formulation with two flavours of quarks with degenerate masses.

Lattice QCD is established on a Euclidean space-time lattice of size $N^{3} \times T$. With lattice spacing set to unity, a point $x$ on the lattice has integer coordinates $x=\left(t, x_{1}, x_{2}, x_{3}\right)$, which are in the range $0 \leq t<T ; 0 \leq$ $x_{i}<N$. A gauge field $U_{x, \mu} \in S U(3)$ is assigned to the link pointing from point $x$ to point $(x+\mu)$, where $\mu=0,1,2,3$ designates the four forward directions in space-time. In this study, periodic boundary conditions 
have been taken for the gauge fields and for the quark fields in all four directions. The full partition function for Wilson QCD is given by

$$
\mathcal{Z}=\int \mathcal{D} U \exp \left(-S_{g}\right) \operatorname{Det}\left(Q^{2}\right)=\int \mathcal{D} U \mathcal{D} \phi^{\dagger} \mathcal{D} \phi \exp \left(-S_{g}-\phi^{\dagger} Q^{-2} \phi\right) .
$$

The term $S_{g}$ in the exponential is the pure gauge action and is given by

$$
S_{g}=-\frac{\beta}{6} \sum_{P} \operatorname{Tr}\left(U_{P}+U_{P}^{\dagger}\right) .
$$

The symbol $U_{P}$ represents the usual plaquette term on the lattice. The determinant factor $\operatorname{Det}\left(Q^{2}\right)$ represents the contribution of internal fermion loops to the theory. The matrix $Q$ that appears in the determinant is a Hermitian sparse matrix defined by:

$$
Q(U)_{x, y}=c_{0} \gamma_{5}\left[\delta_{x, y}-\kappa \sum_{\mu}\left(1-\gamma_{\mu}\right) U_{x, \mu} \delta_{x+\mu, y}+\left(1+\gamma_{\mu}\right) U_{x-\mu, \mu}^{\dagger} \delta_{x-\mu, y}\right],
$$

with $\kappa$ the so-called hopping parameter and $c_{0}=1 /(1+8 \kappa)$. The aim of the simulation is to generate configurations according to the probability distribution $\exp \left(-S_{\text {eff }}\right) \equiv \exp \left(-S_{g}-\phi^{\dagger} Q^{-2} \phi\right)$ using Monte Carlo methods.

The Hamiltonian for the simulations of lattice QCD is given by

$$
\mathcal{H}=\sum_{x, \mu} \frac{1}{2} \operatorname{Tr}\left(H_{x, \mu}^{2}\right)+S_{\text {eff }}\left(U_{x, \mu}, \phi^{\dagger}, \phi\right),
$$

where $H_{x, \mu}$ is the momentum conjugate to the gauge field $U_{x, \mu}$ and takes values in $s u(3)$, the Lie algebra of $S U(3)$.

Since the bosonic part $S_{b} \equiv \phi^{\dagger} Q^{-2} \phi$ is quadratic in the $\phi$ fields, these are generated at the beginning of each molecular dynamics trajectory via $\phi=Q R$, where $R$ is a random spinor field which is Gaussian distributed. The kinetic term in eq. (5) is also generated from Gaussian noise at the beginning of the update. Then the gauge fields and their corresponding momenta are updated according to the equations of motion:

$$
\dot{U}_{x, \mu}=i H_{x, \mu} U_{x, \mu}, \quad i \dot{H}_{x, \mu}=\left[U_{x, \mu} F_{x, \mu}\right]_{T . A .},
$$

where the symbol $[\cdots]_{T . A}$. stands for taking the traceless anti-Hermitian part of the matrix [9] and the quantity $U_{x, \mu} F_{x, \mu}$ is the total force associated with the link $U_{x, \mu}$. The dot on a field variable represents the derivative with respect to the Monte Carlo time $\tau$. The quantity $F_{x, \mu}$ is 
nothing but the "coefficient" in the change of the effective action when an infinitesimal change of the gauge link $\delta U_{x, \mu}$ is applied, i.e.

$$
\delta S_{\text {eff }}=\sum_{x \mu} \operatorname{Tr}\left(F_{x, \mu} \delta U_{x, \mu}+F_{x, \mu}^{\dagger} \delta U_{x, \mu}^{\dagger}\right) .
$$

One can easily check that the time evolution described by eqs. (6) conserve the Hamiltonian (5). Equations (6) define a Hamilton flow in a phase-space manifold that is a direct product of $4 N^{3} T$ factors of $S U(3)$ and $s u(3)$.

Equations (6) establish the molecular dynamics part of the HMC algorithm and it is in this part where the problems with roundingerrors appear. Note that one considers here a purely classical set of autonomous first-order differential equations.

\section{Liapunov exponents}

In this section, we briefly describe the concept of the Liapunov characteristic exponents [10, 11, 12]. Liapunov exponents serve as an important quantitative measure for the degree of stochasticity of a dynamical system. For an introduction to this topic, see [13] and references therein.

Let us consider a time evolution $q(\tau), p(\tau)$, described by a set of firstorder autonomous differential equations like eqs. (1). To each point in the phase-space manifold $(q, p)$ one can attribute a local Liapunov exponent. These exponents describe the mean exponential rate of divergence of the distance between the trajectories of two nearby points in phase-space. Given two points in the phase-space manifold, which are close to each other at $\tau=0$, one can follow the time evolutions of the trajectories originating from these two points. At any given instance of the time $\tau$, one can construct the vector pointing from a point on one of the trajectories to the corresponding one on the other trajectory. If the distance between the two initial points in phase-space becomes infinitesimally small, the above-mentioned vector belongs to the tangent space of the phase-space manifold.

The Liapunov exponents can now be determined by studying the time evolution of these tangent vectors. In order to be specific, we will directly discuss the example of the equations of motion used in the HMC algorithm as applied for lattice QCD. We start denoting by $d H_{x, \mu}$ 
and $d X_{x, \mu}=-i U_{x, \mu}^{-1} d U_{x, \mu}$ the tangent vectors of $H_{x, \mu}$ and $U_{x, \mu}$, respectively. The time evolution of these vectors is described by

$$
\dot{d X_{x, \mu}}=U_{x, \mu}^{-1} d H_{x, \mu} U_{x, \mu}, \quad i \dot{H}_{x, \mu}=d\left[U_{x, \mu} F_{x, \mu}\right]_{T . A .},
$$

which follow from eqs. (6). One can study this set of equations for $\left(d H_{x, \mu}, d X_{x, \mu}\right)$ together with the original eqs. (6). Note that eqs. (8) are linear in $d H_{x, \mu}$ and $d X_{x, \mu}$, both of which are elements of $s u(3)$. Let us now assume that, initially at $\tau=0,\left(d H_{x, \mu}, d X_{x, \mu}\right)$ were given some value in the tangent space of some point in the phase-space manifold. We can introduce the norm in the tangent space as:

$$
D^{2}(\tau)=\sum_{x, \mu} \operatorname{Tr}\left(d H_{x, \mu}^{2}(\tau)+d X_{x, \mu}^{2}(\tau)\right) .
$$

As time evolves, this norm will change and the Liapunov exponent can be defined as:

$$
\nu=\lim _{D(0) \rightarrow 0} \lim _{\tau \rightarrow \infty} \frac{1}{\tau} \log \frac{D(\tau)}{D(0)} .
$$

In fact, due to its linear nature in $\left(d H_{x, \mu}, d X_{x, \mu}\right)$, eqs. (8) can be written as

$$
\frac{d}{d \tau}\left(\begin{array}{c}
d H_{x, \mu} \\
d X_{x, \mu}
\end{array}\right)=J\left[H_{x, \mu}, U_{x, \mu}\right] \cdot\left(\begin{array}{c}
d H_{x, \mu} \\
d X_{x, \mu}
\end{array}\right),
$$

and the Liapunov exponents are just the time averages of eigenvalues of the matrix $J$ along a trajectory. These exponents can be ordered according to the magnitude of their real part. The one with the largest real part is called the leading Liapunov exponent and will be studied in more detail below. It is known [11] that Liapunov exponents do not depend on the choice of metric in phase-space.

The numerical calculation of Liapunov exponents of a given flow can be done straightforwardly [12]. We now go to the discretized versions of of eqs. (6) and eqs. (8) and integrate them simultaneously in time using a leap-frog integration scheme. As a safety measure, in order to avoid severe rounding-errors, the tangent vector should be renormalized with respect to its initial norm after each integration step. The procedure goes as follows: Starting at $\tau=0$, we have some initial value of $D^{2}(0)=$ $\sum_{x, \mu} \operatorname{Tr}\left(d H_{x, \mu}^{2}+d X_{x, \mu}^{2}\right)$ for a given initial tangent vector $\left(d H_{x, \mu}, d X_{x, \mu}\right)$. We

*In the discretized version of numerical integration, we replace the differentials by the corresponding differences. 
then integrate one step in time with step size $\delta \tau$ using the leap-frog scheme. Now, we evaluate the norm $D(\delta \tau)$ of the new tangent vector and store this information for the computation of the Liapunov exponents. Next, we rescale the new tangent vector in such a way that its norm is still equal to $D(0)$, i.e. $\left(\overline{d H}_{x, \mu}(\delta \tau), \overline{d X}_{x, \mu}(\delta \tau)\right)=\left(d H_{x, \mu}(\delta \tau), d X_{x, \mu}(\delta \tau)\right) / D(\delta \tau)$. We then proceed to the next integration step, starting from this already normalized tangent vector. Because of the linear nature of eqs. (8), this rescaling is legitimate since the Liapunov exponents only depend on the ratio of the norms. We then proceed to integrate over $n$ such steps. It can be shown [12] that the average

$$
\nu_{n}=\frac{1}{n \delta \tau} \sum_{k=1}^{n} \log \frac{D(k \delta \tau)}{D(0)}
$$

is approaching the leading Liapunov exponent when $n \rightarrow \infty$. In addition, its value is independent of the value of $\delta \tau$, as long as the step size $\delta \tau$ is not too large.

In contrast to the truly classical Hamilton flows, for which the phase space is usually partitioned into regular and stochastic regions, in the case of the HMC algorithm considered here, the exponent we find is a phase space average of local exponents weighted with the appropriate Boltzmann factor.

Another remark is that the concept of Liapunov exponents could be generalized to higher-dimensional objects as well; not surprisingly, the leading exponent for a $p$-dimensional volume formed by $p$ linear independent tangent vectors is the sum of the $p$ leading exponents of the tangent vector. We point this out because this observation can serve as a tool for calculating the subleading Liapunov exponents if one wishes to.

\section{Liapunov exponent for simulations of $Q C D$}

We have used the method described in the previous section to study the Liapunov exponents in simulations of QCD. A determination of the leading Liapunov exponent for QCD simulations with gauge group $S U(2)$ was reported in [3]. Similar studies were done for gauge group $S U(3)$ in [曰, 60]. Here, we would like to extend these studies by computing the Liapunov exponents in a range of coupling parameter values. 
When numerically integrating the equations of motion derived from the lattice QCD action, we have chosen a step size of $\delta \tau=0.02$, which results in an acceptance of almost $100 \%$. We have integrated the equations of motion with 200 steps, which amounts to a trajectory length of 4.0. The calculations are mainly done on a $4^{4}$ lattice but we also used $8^{4}$ and $16^{4}$ lattices. We checked that our results do not depend on the value of the step size for $\delta \tau \leq 0.02$. The initial values of $\left(d H_{x, \mu}, d X_{x, \mu}\right)$ are generated from a Gaussian distribution. Then the norm of the tangent vector is recorded for each time step, from which we could define the "instantaneous" Liapunov exponent at the $k$-th step as:

$$
\nu(\tau)=\frac{1}{\delta \tau} \log \frac{D(\tau)}{D(0)}, \quad \tau=k \delta \tau .
$$

Note that, as discussed above, in the actual simulation $D(\tau)$ is always normalized to the norm of the starting vector in order to avoid potential rounding-errors. In order to monitor the change of $d H_{x, \mu}$ with $\tau$ and $d X_{x, \mu}$ separately, we have also recorded the values of $D_{H}(\tau)$ and $D_{X}(\tau)$, which are defined as

$$
D_{H}^{2}(\tau)=\sum_{x \mu} \operatorname{Tr}\left(d H_{x, \mu}^{2}(\tau)\right), \quad D_{X}^{2}(\tau)=\sum_{x \mu} \operatorname{Tr}\left(d X_{x, \mu}^{2}(\tau)\right) .
$$

We also define the "instantaneous" -or effective- exponent for $d H_{x, \mu}$ as:

$$
\nu_{H}(\tau)=\frac{1}{\delta \tau} \log \frac{D_{H}(\tau)}{D_{H}(0)},
$$

and similarly $\nu_{X}(\tau)$ for $d X_{x, \mu}$. Again we remark that $D_{H}(\tau)$ receives a proper normalization in the simulation.

We first describe our results for the Liapunov exponents obtained in pure $S U(3)$ gauge theory on $4^{4}$ lattices. We have studied the theory for various values of the gauge coupling $\beta$ ranging from $\beta=0.5$ up to $\beta=30$. In fig. [1, we show the behaviour of the instantaneous Liapunov exponents as a function of the trajectory length for various values of $\beta$. From these figures, it is clearly seen that the exponents show an oscillatory behaviour, especially at the beginning of a trajectory. Then the amplitude of the oscillation dies out as time evolves. Finally, all three exponents $\nu, \nu_{H}$ and $\nu_{X}$ approach the same stable constant value. The leading Liapunov exponent can be extracted by filtering out the zero frequency part in $\nu(\tau)$ towards the end of the trajectory. In our determination of the leading exponent, we fit the tail of the function 

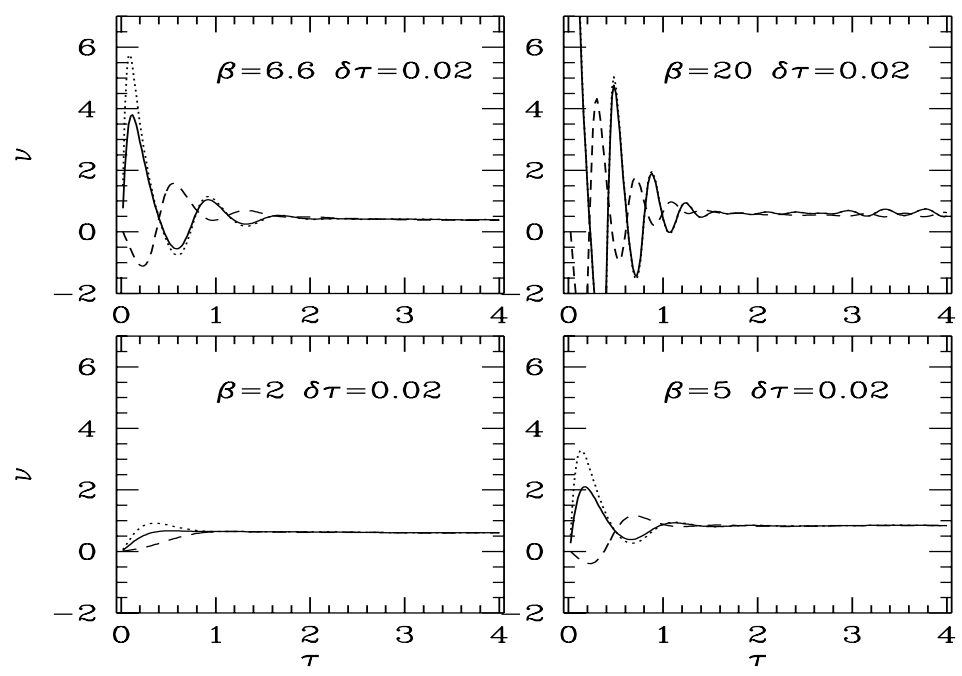

Figure 1: The instantaneous Liapunov exponents, as defined in eq. (13) and eq. (15), are plotted as a function of the Monte Carlo time $\tau$ along a trajectory for various values of $\beta$. The dotted lines represent $\nu_{H}(\tau)$ while the dashed lines represent $\nu_{X}(\tau)$.

The $\nu(\tau)$ 's are represented by solid lines.

$\nu(\tau)$ to a constant. We also note that as the value of $\beta$ is increased, so is the frequency and magnitude of the oscillation. This is a reflection of the common expectation that for large values of $\beta$, the system is getting closer to a Gaussian model. Despite rather strong oscillations for $\nu(\tau)$ at large values of $\beta$, the integral $R(\tau)$ of the function $\nu(\tau)$, defined as

$$
R(\tau)=\sum_{j=1}^{k} \delta \tau \nu(j \delta \tau), \quad \tau=k \delta \tau,
$$

which reflects the change in the norm relative to the starting point, is basically increasing linearly with only little oscillations, as shown in fig. 2.

Having extracted the leading Liapunov exponents for for several values of $\beta$, we plot them as a function of $\beta$ in fig. 3 (a). We see from this figure that the Liapunov exponents show a significant $\beta$-dependence.

${ }^{\dagger}$ Similar studies have also been done by the authors of ref. [6. Complete consistent values of the exponent have been obtained, except for very large $\beta$ values. 

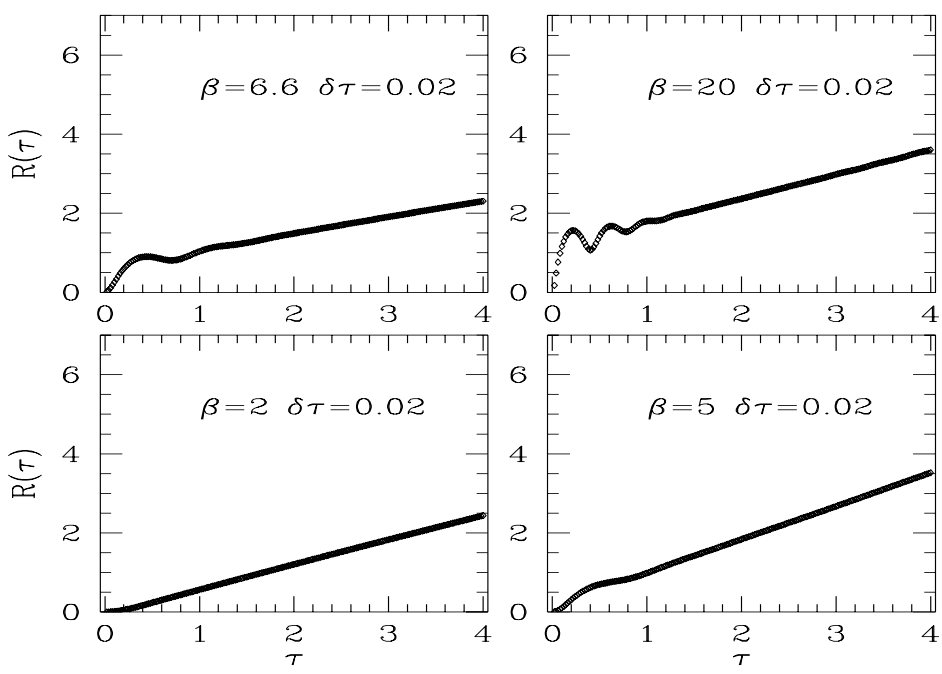

Figure 2: The integral of the function $\nu(\tau)$, eq. (16), which represents the change in the norm of the tangent vector as compared to its original value, is plotted as a function of the Monte Carlo time of a trajectory for the same values of $\beta$ as in fig. 1.

From $\beta=5.0$ to $\beta=10.0$, we have a leading Liapunov exponent that ranges from 0.85 to 0.35 .

In fig. B(a), we plot also results for the Liapunov exponents for an $8^{4}$ (filled triangles) and for a $16^{4}$ lattice (stars). We can see that the Liapunov exponents for the different size lattices fall essentially on one common curve. This behaviour is not in accord with the anticipated property of the Liapunov exponent to be inversely proportional to the correlation length, as proposed in [6]. The data seem to indicate that we are really studying here classical equations of motion derived from the 4-dimensional classical Hamiltonian given in eq. (5).

We have also performed similar investigations of the Liapunov exponents for full QCD with dynamical fermions. In dynamical QCD simulations, the parameter $\kappa$ appears and the value of the Liapunov exponent, in principle, will also depend on this parameter. For $\kappa$ values that we have studied on $4^{4}$ lattices, the leading Liapunov exponent shows, however, only a rather weak dependence on $\kappa$.

We have performed a rough scan in the parameter ranges $5.6 \leq \beta \leq 8.0$ and $0.13 \leq \kappa \leq 0.16$. The Liapunov exponents are measured in the same 


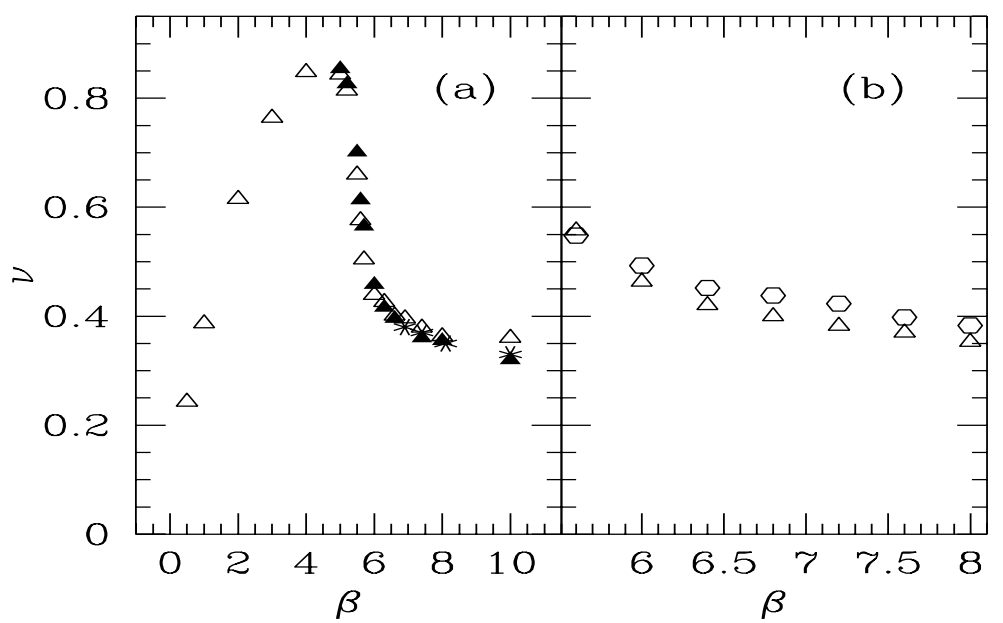

Figure 3: The average leading Liapunov exponents for pure $S U(3)$ gauge theory (a) and full QCD (b) as a function of the gauge coupling $\beta$. In (a), the open and filled triangles correspond to $4^{4}$ and $8^{4}$ lattices, respectively. The stars denote our results for a $16^{4}$ lattice. In (b), exponents for different values of $\kappa$ are represented by different symbols. The triangles correspond to $\kappa=0.13$ and the hexagons to $\kappa=0.16$.

way as in the pure gauge case. The instantaneous exponents also show similar behaviours. In fig. 3(b), we show the final result of the exponents as a function of $\beta$ and $\kappa$. Different $\kappa$ values for the same $\beta$ give almost the same value for the Liapunov exponents. For each value of $\beta$, we have taken four different values of $\kappa$, namely $0.13,0.14,0.15$ and 0.16 . Due to their weak dependence on $\kappa$, we only show the exponents for two values of $\kappa$, i.e. $\kappa=0.13$ (triangles) and $\kappa=0.16$ (hexagons). The errors of the points are about the size of the symbols. In the parameter range that we have studied, the Liapunov exponent is roughly $0.4-0.5$.

\section{Consequences of irreversibility}

The equations of motion in eqs. (6) are to be reversible to ensure the exactness of the HMC algorithm used for the simulations of lattice QCD. However, rounding-errors in the numerical integration process of this algorithm imply violations of the reversibility condition. Thus, the exactness of the algorithm can no longer be guaranteed. Although 
this brings us into an unsatisfactory situation from a principle point of view, it is necessary to investigate what are the consequences of the reversibility violations.

For a molecular dynamics trajectory there are two causes for an increase of rounding-errors when the trajectory length $\tau$ is increased for fixed integration step size $\delta \tau$. If we assume that the rounding-errors are Gaussian distributed, we expect a random walk behaviour and hence that the rounding-errors grow like $\sqrt{\tau}$. Second, as demonstrated above and also found in [3, $4,5,6$, since the equations of motion behave like those of a classical chaotic system, the rounding-errors get amplified exponentially, like $\exp (\nu \tau)$, with a positive Liapunov exponent $\nu$.

Both effects lead to a certain amount of reversibility violation and in this section we try to quantify the effect of this reversibility violation on physical observables on the one hand and on the Hamiltonian that enters the Metropolis decision in the HMC algorithm on the other hand.

\subsection{Observables}

To study the effects of the above discussed reversibility violation on observables, we have run two HMC simulation programs in parallel. One of them is with 32-bit arithmetic but with 64-bit arithmetic scalar products and summations over the lattice, which mimics the typical situation in simulations on a 32-bit machine. The other one runs with complete 64-bit arithmetic and serves as a reference point for an "exact" program. Let us remark that for this section we have chosen $S U(2)$ as the gauge group.

We then proceeded as follows: one generates a configuration, say with the 64-bit arithmetic program version. On this configuration one starts the 32-bit and the 64-bit program versions and run them for a given number of molecular dynamics steps with a fixed step size, i.e. up to some trajectory length $\tau$. At each step of the trajectory, one measures quantities such as the plaquette $P$, the Polyakov line $L$, plaquette correlation functions or, in the case of dynamical fermions, the scalar density. Let us denote such a quantity with $O$ for the 64-bit version of the program and with $\tilde{O}$ the corresponding one for the 32-bit

arithmetic version. As an example, let us give the measurement of the 
Polyakov line:

$$
L_{\vec{x}}=\frac{1}{2} \operatorname{Tr} \prod_{t=0}^{T-1} U_{(\vec{x}, t), 0},
$$

i.e. the trace of the ordered products of gauge field variables. The index $\vec{x}$ denotes the 3-dimensional space coordinate. We then measure at each molecular dynamics step

$$
\|L-\tilde{L}\|^{2}=\frac{1}{N^{3}} \sum_{\vec{x}}\left(L_{\vec{x}}-\tilde{L}_{\vec{x}}\right)^{2} .
$$

For other observables the difference $\|O-\tilde{O}\|$ can be computed in an obviously generalized analogous way. The whole procedure is repeated on a number of configurations in order to obtain an error estimate. From the above discussion we expect that

$$
\|O-\tilde{O}\|=c_{0}+c_{1} \sqrt{\tau}+c_{2} e^{\nu \tau} .
$$

The result for the Polyakov line on a $6^{3} 8$ lattice is shown in fig. 4, together with a fit to the data according to eq. (19) for both the pure gauge theory (open squares) and dynamical fermion simulations (filled squares). Obviously, the fit formula eq. (19) provides an excellent description of the data. We checked that also the other above-mentioned observables can be fitted by eq. (19). We remark that for the scalar density the exponential growth of rounding-errors sets in for only large trajectory lengths, $\tau \approx 8$. We conclude that indeed both sources of rounding-errors, the random walk and the chaotic behaviour, seem to be present in our simulations in practice and show up in physical observables.

We find the same rounding-error behaviour of eq. (19) when we first sum up the observables over the lattice, constructing $O_{g}=\sum_{x} O_{x}$, and then build the global difference $\left|O_{g}-\tilde{O}_{g}\right|$ at each molecular dynamics step. The prominent exception is the global difference of the plaquette, $\mid P_{g}-$ $\tilde{P}_{g} \mid$, which shows an oscillatory behaviour similar to the ones shown in fig. 1. The reason for these oscillations is that in the HMC algorithm the total Hamiltonian is, up to finite step size errors, conserved. Therefore, the tangent vector that corresponds to the plaquette has to compensate the oscillations of the tangent vector that corresponds to the momenta studied in section 4.

For a lattice size of $6^{3} 8$ we see from fig. (4) that the difference $\|L-\tilde{L}\|$, which indicates the deviation from the true value, is very small and hard 


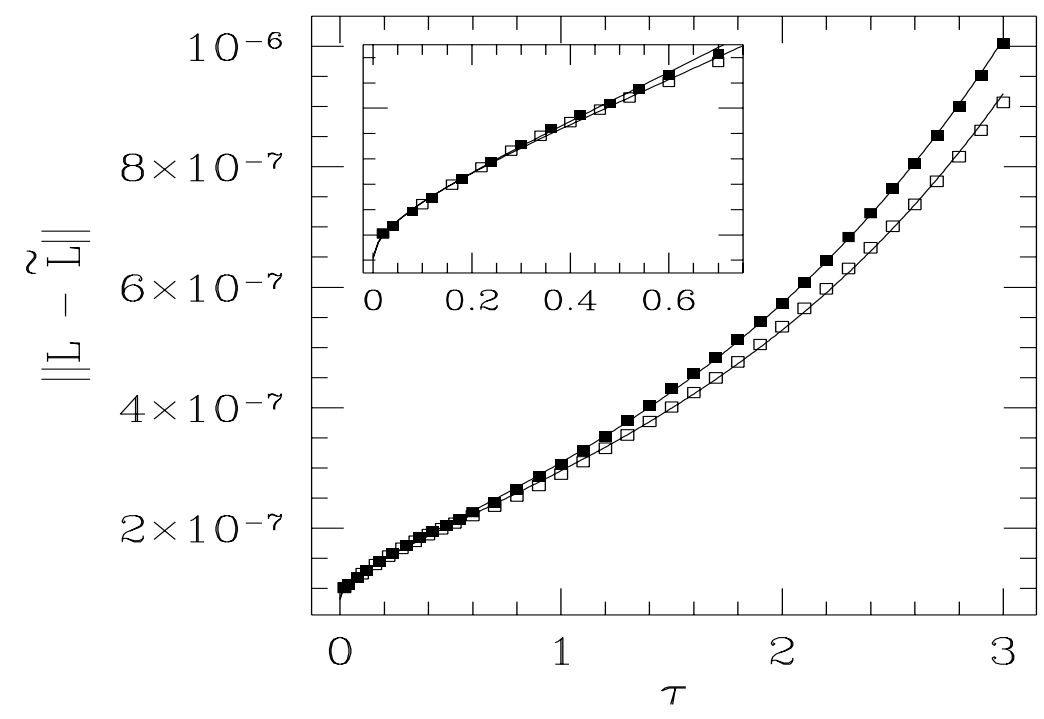

Figure 4: The difference $\|L-\tilde{L}\|$, eq. (18), for the Polyakov line $L$ as a function of the trajectory length $\tau$. Open squares are for the pure gauge theory at $\beta=2.12$ and filled squares are from full dynamical fermion simulations at $\beta=2.12, \kappa=0.15$. The small inset amplifies the region of small $\tau$. The solid line is a fit according to eq. (19).

to detect in a simulation. By true value we here mean -by definitionthe value of the observables from the 64-bit arithmetic program version. It is only for very long trajectories that one would risk the danger to produce significant differences.

One may expect that the coefficients $c_{i}$ in eq. (19) depend on the lattice size. Our original plan was therefore to extract the volume dependence of these coefficients and to extrapolate to a lattice size, where rounding-error effects and in particular the consequences of irreversibility will become dangerous for trajectories of length 1. Our findings for

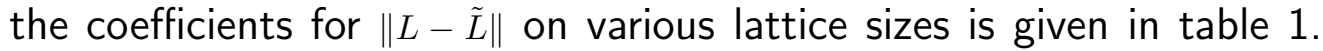
To our surprise, the fit parameters show basically no lattice size dependence at all. A similar finding was obtained for the plaquette variable and for plaquette correlation functions at various distances. 
Table 1: Lattice dependence of the fit parameters in eq. (19) for the Polyakov line at $\beta=2.12$ for the quenched case and $\beta=2.12, \kappa=0.15$ for the unquenched simulations.

\begin{tabular}{clllll}
\hline Lattice & & $c_{1} \cdot 10^{6}$ & $c_{2} \cdot 10^{6}$ & \multicolumn{1}{c}{$\nu$} & $c_{0} \cdot 10^{6}$ \\
\hline \hline $4^{3} 8$ & quenched & $0.1180(10)$ & $0.108(2)$ & $0.630(6)$ & $-0.027(2)$ \\
$6^{3} 8$ & quenched & $0.1177(6)$ & $0.104(1)$ & $0.654(3)$ & $-0.023(1)$ \\
$8^{4}$ & quenched & $0.1177(6)$ & $0.103(1)$ & $0.659(3)$ & $-0.022(1)$ \\
$4^{3} 8$ & dynamical & $0.1111(14)$ & $0.143(4)$ & $0.557(8)$ & $-0.062(4)$ \\
$6^{3} 8$ & dynamical & $0.1066(21)$ & $0.143(5)$ & $0.608(10)$ & $-0.060(5)$ \\
$8^{4}$ & dynamical & $0.1115(17)$ & $0.134(4)$ & $0.623(8)$ & $-0.052(4)$ \\
\hline
\end{tabular}

For the HMC algorithm applied for the pure gauge theory one may give a simple reasoning: since in the HMC algorithm the gauge links only feel their nearest neighbours, their update is essentially local. But, for dynamical fermions, in the equations of motion the information of the inverse fermion matrix is used, which is non-local. The results of table 1 are therefore somewhat counter-intuitive. This indicates that the difference between the 32-bit and 64-bit versions in the solution vectors obtained from the conjugate gradient (CG) method do not increase significantly with the volume. We checked this explanation explicitly by studying the stability of the CG inversion method against roundingerrors. In addition it might be that our studies are performed in a situation where the propagators vanish fast with growing distance. Therefore information would only be transported over a few lattice spacings.

\subsection{Hamiltonian}

Would the HMC algorithm only contain the molecular dynamics part, the results of the previous section would indicate that rounding-error effects show up in physical observables only for a very large number of integration steps. However, the exactness of the HMC algorithm requires a Metropolis reject/accept step for which the difference of the initial and final values of the Hamiltonians are taken. An important observation is that for this difference an absolute precision is required. The computations of the acceptance Hamiltonians therefore play a special role and an investigation of rounding-error effects on them is most 
crucial.

We therefore measured in full QCD the difference between the value of the Hamiltonian $\tilde{H}$ from the 32-bit arithmetic program version and the one from the 64-bit arithmetic program version $H$. Let us define the ratio

$$
R_{H}=\frac{\langle|\delta(\Delta H)|\rangle}{\langle|\Delta H|\rangle},
$$

where $\langle|\delta(\Delta H)|\rangle=\langle|\Delta \tilde{H}-\Delta H|\rangle$ and $\Delta H$ is the difference between the initial Hamiltonian at the beginning and the final Hamiltonian evaluated at the end of a trajectory. One should expect a dependence on the lattice size for $R_{H}$. Keeping the acceptance rate constant means that $\langle|\Delta H|\rangle$ should be constant. However, the values for $H$ themselves increase substantially with the lattice size since they are constructed by a sum over all lattice points, see eq. (5). As a consequence, the rounding-errors become more and more significant relative to $\langle|\Delta H|\rangle$.

Indeed, averaging over 100 trajectories, we find $R_{H} \approx 0.08 \%, 0.18 \%$ and $0.70 \%$ for $8^{4}, 12^{4}$ and $16^{4}$ lattices, respectively. These numbers are obtained by performing only 10 molecular dynamics steps with step size $\delta \tau=0.02$, a number that is certainly too small for simulations on a $16^{4}$ lattice. Still, extrapolating $R_{H}$ linearly in $N^{2}$ to a $32^{4}$ lattice we find that $\langle|\delta(\Delta H)|\rangle$ could become about $5 \%$ of $\langle|\Delta H|\rangle$. We think that this number is a conservative estimate of the rounding-error effects on the acceptance Hamiltonian on such a large lattice. We feel that performing simulations on lattices of comparable sizes on 32-bit arithmetic computers could not be safe. The same holds, of course, on 64-bit arithmetic machines when 32-bit arithmetics is used for the numerical computations.

\section{Conclusions}

In this paper, we have investigated several questions related to the reversibility problem of the molecular dynamics kind of algorithms as used for the simulation of lattice QCD. The purely classical equations of motion used in these algorithms behave like those of a chaotic dynamical system. We have determined the leading Liapunov exponents $\nu$ for both pure $S U(3)$ gauge theory and full QCD for various bare parameters. We have found a weak dependence of the leading Liapunov exponent on the hopping parameter $\kappa$, whereas the $\beta$-dependence is more significant. We argued that, due to the chaotic nature of the equations of motion, 
the rounding-error that occurs in the integration of these equations is magnified exponentially with increasing trajectory length, when keeping the step size constant.

We suggested that the growth of the rounding-errors as a function of the trajectory length $\tau$ should consist of two parts: the first is a random walk behaviour, leading to a $\sqrt{\tau}$ part. The second is the exponential amplification, leading to an $\exp (\nu \tau)$ contribution. We verified that this ansatz is indeed followed by observables such as the plaquette, the Polyakov line and plaquette correlation functions. Considering the above observables, the rounding-errors, however, do not increase with the lattice size, at least for the parameter values we considered here. The situation is different for the value of the difference $\Delta H$ of the initial and final Hamiltonians, computed at the beginning and the end of a molecular dynamics trajectory, which enters the Metropolis decision in the HMC algorithm. This quantity plays a special role in the molecular dynamics kind of algorithms, because it is the absolute precision with which these Hamiltonians have to be calculated. We find that, with growing lattice size, the rounding-errors for $\Delta H$ increase. Extrapolating the rounding-error effects as found on $8^{4}, 12^{4}$ and $16^{4}$ lattices to a lattice of size $32^{4}$, gives a rounding-error for $\Delta H$ that can reach several per cent. We regard this estimate as rather conservative and therefore conclude that simulations on lattices of size $32^{4}$ using 32-bit arithmetics, either by hardware or by software, could be dangerous.

A real test of the effects of rounding-errors on observables would be to run a 32-bit arithmetic program version against a 64-bit arithmetic version for a long time and see, whether one finds differences in some observables. Here we could only perform approximations of this test. We can therefore not give a definite answer of whether rounding-errors will lead to problems for the molecular dynamics kind of algorithms. However, our data indicate that it can be dangerous to use these algorithms for simulating large lattices employing 32-bit arithmetics.

\section{Acknowledgements}

We would like to thank $M$. Lüscher for his critical reading of the paper. We thank members of the SESAM collaboration for useful discussions. Critical comments on our draft by A. Frommer, I. Horvath, A. D. Kennedy and R. Sommer are gratefully acknowledged. 


\section{References}

[1] S. Duane, A.D. Kennedy, B.J. Pendleton and D. Roweth, Phys. Lett. B195 (1987) 216.

[2] A. M. Horowitz, Phys. Lett. 156B (1985) 89; Nucl. Phys. B280 (1987) 510; Phys. Lett. 268B (1991) 247.

[3] K. Jansen and C. Liu, Nucl. Phys. B 453 (1995) 375 and B 459 (1996) 437.

[4] U. Glässner, S. Güsken, H. Hoeber, T. Lippert, X. Luo, G. Ritzenhöfer, K. Schilling and G. Siegert, hep-lat/9510001.

[5] R. C. Brower, T. Ivanenko, A. R. Levi and K. N. Orginos, Nucl. Phys. B484 (1997) 353.

[6] R. G. Edwards, I. Horvath, A. D. Kennedy, Nucl. Phys. B484 (1997) 375.

[7] R. Edwards and A.D. Kennedy, private communications.

[8] K. Jansen and C. Liu, Nucl. Phys. B (Proc.Suppl.) 53 (1997) 974.

[9] S. Gottlieb, W. Liu, D. Toussaint, R. L. Renken and R. L. Sugar, Phys. Rev. D 35 (1987) 2531.

[10] A. M. Liapunov, Ann. Math. Studies 17 (1907) 1947.

[11] V. I. Oseledec, Trans. Moscow Math. Soc. 19 (1968) 197.

[12] G. Benettin, L. Galgani and J. M. Strelcyn, Phys. Rev. A14 (1976) 2338.

[13] A. J. Lichtenberg and M. A. Lieberman, Regular and Chaotic Dynamics, 2nd ed., Springer-Verlag, 1992. 